\title{
Dispositivos Lúdico - Pedagógicos para desarrollar habilidades de resolución de conflictos escolares
}

\section{Playful - Pedagogical devices to develop school conflict resolution skills}

\author{
Edgar Augusto Chevel Herrera \\ Magister en Educación \\ Institución Educativa San Clemente \\ Colombia \\ edgarachh@gmail.com
}

\author{
Fernando Antonio De La Espriella Arenas \\ Magister Educación y Desarrollo Humano \\ Universidad de Córdoba \\ Colombia \\ fdelaespriella@correo.unicordoba.edu.co
}

\section{Resumen}

Palabras claves:

Conflictos en el aula, Convivencia escolar, Dispositivos lúdico pedagógicos, Educación, Formación, Interacción en el aula.
La escuela es un dispositivo social cuya función es formar sujetos que estén en condiciones de participar de manera adecuada en las dinámicas de la vida social. No obstante, estas funciones se ven impactadas por una serie de variables que le afectan en todos los ámbitos de su desarrollo: uno de ellos, es el conflicto escolar. La presente comunicación devela los resultados de investigación en la cual se aborda el conflicto escolar y se implementan dispositivos lúdico-pedagógicos (DLP), como forma de intervenir esta realidad. Por ello, se formuló como objetivo implementar desde la Educación Física, Recreación y Deporte dispositivos lúdico - pedagógicos encaminados a la transformación y resolución de conflictos de convivencia escolar en el grado cuarto de la Institución Educativa San Clemente de Tierralta - Córdoba. En su estructura interna, sigue la senda de la investigación cualitativa, con una metodología enfocada desde la investigación acción participación (IAP). En ella se abordan diferentes técnicas de investigación que van desde entrevistas, grupos de discusión, análisis de documentación, diario de campo, observación directa, entre otros. Los resultados se valoran a partir de la voz de los estudiantes, docentes y padres de familia que integraron la investigación, no como objetos, sino como sujetos de esta. En esta pesquisa se le otorga valor a la reflexión y auto reflexión de los sujetos participantes, es a parir de ella como se construye teoría y valida la intervención. Como resultados se presenta un impacto positivo en las relaciones interpersonales de los estudiantes -valorados desde sus reflexiones- y la adopción de los DLP como modelo para el tratamiento de los conflictos escolares en la institución educativa.

Recepción: Diciembre 09 de 2019 | Aceptación: Marzo 13 de 2020 | Publicación: Junio 30 de 2020 


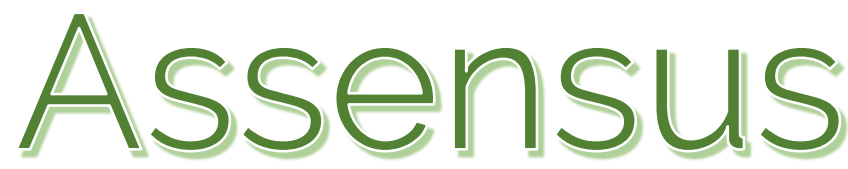

Revista de Investigación educativa y pedagógica

\section{Abstract}

Keywords:

Conflicts in the classroom, School coexistence, Playful pedagogical devices, Education, Training, Interaction in the classroom.
The school is a social device whose function is to train subjects who are able to participate adequately in the dynamics of social life. However, these functions are impacted by a series of variables that affect them in all areas of their development: one of them is school conflict. The present communication reveals the research results in which the school conflict is addressed and playful-pedagogical devices (PPD) are implemented, as a way to intervene this reality. For this reason, it was formulated as an objective to implement from the Physical Education, Recreation and Sports playful-pedagogical devices aimed at the transformation and resolution of school coexistence conflicts in the fourth grade of the Institución Educativa San Clemente of Tierralta - Córdoba. In its internal structure, it follows the path of qualitative research, with a methodology focused on actionparticipation research (APR). Moreover, different research techniques are addressed, ranging from interviews, discussion groups, documentation analysis, field diary, direct observation, among others. The results are valued from the voice of the students, teachers and parents who integrated the research, not as objects, but as subjects of - it. In this inquiry, value is given to the reflection and self-reflection of the participating subjects, thus the theory is built and the intervention is validated. As results, a positive impact is presented in the interpersonal relationships of the students -valued from their reflections- and the adoption of the PPD as a model for the treatment of school conflicts in the educational institution.

Received: December 09, 2019 | Accepted: March 13, 2020 | Published: June 30, 2020 


\section{Introducción}

Las situaciones o hechos de conflicto al interior de las Instituciones Educativas (IE) sea cual fuere su naturaleza, son realidades comunes que forman parte de su cotidianidad. Esta situación, de no ser atendida con prontitud y eficiencia puede desbordar la vida cotidiana al interior de las escuelas. Cuando esto sucede, las acciones de violencia y conflicto permean todo el ambiente escolar, generando preocupaciones e incertidumbres en docentes, directivos, padres de familia y estudiantes. De acuerdo al planteamiento anterior, se tiene como ejemplo concreto situaciones conflictivas que se han convertido en actos de violencia al interior de la escuela y en las relaciones de unos y otros actores, entre los cuales se urden una serie de agresiones las cuales podemos entenderlas como: toda acción realizada por una o varias personas que conforman la comunidad educativa y que busca afectar violentamente a otras personas de la misma comunidad, de las cuales por lo menos una es estudiante (Decreto 1965 de 2013, Art. 39). En este orden de ideas, se termina derivando en hechos violentos, entre ellos se pueden destacar el acoso, bullying, intimidación, exclusión, maltrato verbal o físico, entre otros. En la cual, estudiantes, docentes, directivos, padres de familia pueden participar de diferente forma: como agresores, victimas u observadores.

Cuando se aborda el tema del conflicto escolar es necesario consultar datos o reportes que puedan ilustrar su incidencia en el contexto. En ese sentido, el Departamento de Córdoba entre los años 1967 y 2008, fue un escenario donde intervinieron diferentes actores armados que han contribuyeron en la generación de violencia directa o estructural, tales como: guerrillas, narcotráfico, autodefensas y bandas criminales. Todo lo anterior, debido a su posición geográfica y estratégica en el territorio. No obstante, al consultar sobre violencia escolar específicamente, en los entes territoriales -Secretaría de Educación Departamental y municipal, Bienestar Familiar- no existen datos, ni reportes con cifras exactas de los hechos conflictivos y violentos en las IE. Lo anterior, de acuerdo con lo manifestado por funcionarios de estas instancias, se debe a que no se hacen las denuncias respectivas, sin embargo, la realidad es evidente y la preocupación existe.

Este artículo, aborda el conflicto escolar y el contexto de la IE San Clemente sede Nuevo Tay, perteneciente al municipio de Tierralta, en el departamento de Córdoba. La sede principal se encuentra ubicada en la Vereda San Clemente. La ubicación geográfica de la IE, la comunidad asentada en la misma y la realidad social que subyace al entorno escolar, hacen que en la cotidianidad de la escuela se presenten situaciones conflictivas que en muchos casos derivan en hechos violentos entre actores de la comunidad educativa. En dicha institución, se observan algunas situaciones disruptivas que alteran la cotidianidad escolar, discusiones, roces verbales, riñas entre estudiantes, falta interés, poca motivación, entre otros, los cuales no siempre han sido correctamente mediados ni manejados por parte de los docentes, originando episodios de violencia y agresión estudiantil.

En muchos casos, las conductas conflictivas de algunos estudiantes son originadas por el impacto que genera la realidad del contexto en el que ellos se desenvuelven. La afirmación anterior, se deriva del testimonio y experiencias de los docentes de básica primaria de la IE, 
quienes en entrevista abierta expresaron sus apreciaciones y detallaron algunos aspectos específicos relacionados con posibles conflictos en el aula de clase. Las principales dificultades observadas por los docentes en los niños y niñas, y que podrían estar relacionadas con la generación de conflictos escolares son: actitud indiferente, falta de acompañamiento y orientación a los estudiantes por parte de los padres de familia; la intolerancia; exclusión, agresión física o verbal entre los estudiantes; poco respeto a sus semejantes; todo lo anterior ligado al hecho de provenir de hogares disfuncionales, monoparentales y la violencia estructural del entorno.

En consecuencia, el interés de esta investigación surge a partir de las necesidades encontradas en el aula de clases, a través de diferentes diagnósticos y de la vivencia diaria con los estudiantes, donde se han detectado múltiples casos de conflicto escolar los cuales ameritan de manera urgente un estudio profundo y una resolución rápida y efectiva. Así las cosas, esta realidad lleva a indagar en profundidad sobre este tipo de ambientes que rodean a los estudiantes, para con ello, identificar el origen y causa de los conflictos que puedan presentarse y abordarlos con pertinencia, intentado sacar provecho de estos en busca del crecimiento y mejoramiento de las relaciones interpersonales al interior de las instituciones.

De este modo, la investigación estuvo orientada hacia la implementación, desde el área de Educación Física, Recreación y Deporte, de dispositivos lúdico - pedagógicos encaminados a la transformación y resolución de conflictos de convivencia en el grado cuarto de la IE San Clemente de Tierralta - Córdoba. Para ello, fue necesario plantear objetivos específicos que permitieran el propósito del estudio. Inicialmente, se buscó describir el contexto social, comunitario y escolar de la IE san Clemente; luego, identificar los principales elementos, factores y situaciones que conllevan a la generación de conflictos entre los alumnos de grado cuarto; para posteriormente determinar los dispositivos lúdico - pedagógicos a través de los cuales se pudiera intervenir la realidad conflictiva entre los estudiantes del grado cuarto. Todo esto derivó en la operacionalización de dispositivos lúdico - pedagógicos desde la construcción participativa en la Educación Física, Recreación y Deportes que ayudasen a evitar situaciones de conflictos de convivencia entre los estudiantes.

Así mismo, para el reconocimiento del contexto social-comunitario de la IE, se recogieron testimonios de docentes, directivos y padres de familia, que permitieron la identificación de los principales elementos, factores y situaciones que conllevan a la generación de conflictos entre los estudiantes de cuarto grado. Con toda esta información, se procedió a determinar los dispositivos lúdico - pedagógicos, que facilitarían intervenir la realidad conflictiva; en esta fase fue clave la participación de estudiantes, docentes y padres de familia. Finalmente, se da la operacionalización de estos con la constante participación de los diferentes actores de la comunidad educativa.

Para el desarrollo de la investigación se tuvieron en cuenta los planteamientos propuestos en el Proyecto Educativo Institucional -PEI-, desde el cual se pretende formar estudiantes íntegros, lideres, capaces de reconocer su realidad, transformar su comunidad con visión basada en la oportunidad de aprender sobre sus vivencias dentro y fuera del aula, con un acercamiento a la comprensión de diferentes problemáticas. De igual forma, se le dio trascendencia a una problemática que se presenta a nivel regional, departamental, e incluso, 
nacional, debido a las diferentes prácticas de enseñanza o a las políticas educativas que se manejan; sin dejar de mencionar los problemas o formas de aprendizaje de cada niño y niña dentro de las aulas.

En síntesis, se buscó determinar la influencia de los dispositivos lúdico - pedagógicos y la mejora que se puede presentar en las prácticas de los docentes, aprovechando los recursos con que se cuenta en la IE. Por lo cual, se partió de la concientización acerca de la manera que se puede fomentar la convivencia escolar a partir del juego, de la asociación e interpretación de imágenes, y del desarrollo de elementos innovadores que tiendan a afianzar, transformar y poner en práctica los conocimientos adquiridos, todo esto desde una interacción social e intercambio de saberes entre estudiantes y docentes a través de un ejercicio reflexivo, enmarcado desde la Investigación Acción Participativa (IAP).

\section{Referentes teóricos}

Cuando se aborda el tema de convivencia infantil al interior de la escuela, se hace necesario observarlo desde diferentes perspectivas que enriquezcan su tratamiento y reflexión. El presente estudio aborda el concepto de conflicto en clave positiva, de acuerdo con esto, Vinyamata (2015) plantea que el conflicto es una lucha, desacuerdo, incompatibilidad aparente, confrontación de intereses, precepciones entre dos o más partes; en ese sentido, es connatural con la vida misma, ya que está en relación directa con el esfuerzo por vivir. Un elemento importante dentro de este engranaje, es la conflictología expresión acuñada por Galtung (1998) que se entiende como el nexo de colaboración necesario para unir en un esfuerzo de análisis que nos permita comprender las causas de los conflictos y la manera práctica de sustituir los métodos violentos y contradictorios de resolverlos (Vinyamata, 2015).

Por su parte, Merayo (2013) sostiene que el conflicto es un fenómeno propio del quehacer cotidiano puesto que día a día el ser humano está afrontando situaciones que no le convencen, que no le parecen, o sencillamente no son de su agrado, por ende, conllevan a la generación de conflictos los cuales debe procurar abordar de la forma más propicia, pertinente y constructiva posible, independientemente que estemos hablando de un contexto escolar, familiar, laboral o social. Desde postura más abierta, Fisas (2010) sostiene que el conflicto es un proceso interactivo que se da en un contexto determinado; es una construcción social, una creación humana, diferenciada de la violencia. Por tanto, se concluye que el conflicto puede ser positivo o negativo según como se aborde y se termine, con posibilidades de ser conducido, transformado y superado. A continuación, se describen algunas categorías conceptuales que orientaron este estudio. 


\section{Conflicto Escolar}

El conflicto hace parte de la cotidianidad de la escuela y se manifiesta de diferentes formas, al respecto Viñas (2004; citado por García 2015), señala que los conflictos en los centros educativos no son únicamente de un tipo, según las personas que intervengan en el mismo podemos diferenciar cuatro grandes categorías: conflictos de poder, conflictos de relación, conflictos de rendimiento y los conflictos interpersonales. Estas categorías pueden describirse brevemente de la siguiente forma: en los primeros, están comprendidos todos los conflictos referentes a reglas y normas. El segundo tipo de conflictos tiene que ver con el intento de un sujeto de superar jerárquica o emocionalmente al otro. El tercer tipo de conflictos está asociado con las dificultades que puede presentar un estudiante para equilibrar sus necesidades formativas dentro de la institución educativa donde se desenvuelve. Finalmente, el cuarto tipo de conflictos va más allá del campus educativo y tiene que ver con la sociedad que rodea al individuo y la manera en cómo influye sobre éste.

\section{Hostigamiento o bullying}

Esta categoría conceptual se deriva de la anterior y es tan antigua como la escuela misma, solo que hoy se ha hecho más evidente por la consecuencia que ha generado su poca intervención. Gairín, Armengol y Silva (2013) lo definen como aquel comportamiento caracterizado por un seguimiento físico y/o psicológico que ejecuta un ser humano hacia otro. Aquí los autores hablan de un comportamiento agresivo y progresivo, tanto físico como verbal, que el victimario va desarrollando hacia su víctima. Por su parte, Castillo (2011) considera que puede ser visto como cualquier tipo de maltrato o violencia en donde existe un deseo claro de un individuo de lastimar o perjudicar a otro ser más débil.

\section{Convivencia escolar y resolución de conflictos}

Dentro de los aportes teóricos, un concepto que no se debe omitir es el asociado a la convivencia escolar. Esto se debe en mayor medida a que gracias a ella, se permite el desarrollo integral de los niños y jóvenes en su proceso de integración a la vida social, en la participación responsable en la vida ciudadana y en el desarrollo de su propio proyecto de vida. Para Bravo y Herrera (2011) es el resultado elocuente e inevitable de esa necesidad natural que tiene el ser humano de compartir su vida juntos a los demás, iniciando en primera instancia con las relaciones en su núcleo familiar, para pasar más adelante a las relaciones en su comunidad, y posteriormente a las relaciones propias de su entorno escolar. Por ello, abordar el concepto de convivencia en la escuela como parte de formación, se debe posibilitar el desarrollo de diferentes competencias. Así mismo, Guzmán, Muñoz y Preciado (2012), sostienen que puede ser entendida como esa existencia armónica que se desarrolla dentro del colegio, y que además favorece el desarrollo y el proceso de enseñanza - aprendizaje de los niños.

En lo referente al concepto de resolución de conflictos, Calderón (2011) sostiene que, es una técnica de comunicación adecuada, apropiada y pertinente que permite gestionar 
acertadamente la sana convivencia dentro de un contexto escolar determinado. Este sentido se puede inferir que el conflicto es una clara situación que tiende a darse en cualquier institución educativa, y que por lo tanto requiere de una especial atención de los directivos y docentes para una resolución acertada de los mismos.

\section{Dispositivos lúdico-pedagógicos}

De acuerdo con Camilloni (2013) los dispositivos Lúdico-Pedagógicos (DLP), son aquellos diseños que pertenecen a una clase particular de aprendizaje, a una estrategia de enseñanza con enfoque holístico que está destinada a relacionar el aprendizaje académico con la vida real, en donde se proponen al estudiante actividades en las que, a partir de esa conexión con la práctica, se le requiere que ponga a prueba sus aprendizajes en situaciones auténticas. El DLP funciona como un dispositivo de resistencia y potenciación diseñado para provocar cambios, transformaciones individuales y colectivas. Para su diseño e implementación es necesario echar mano del juego, el arte, la creatividad, la música, el baile; todas las anteriores como medios que hacen de su creación un acto original que estimula emociones, sentidos y sentimientos en todos los participantes.

Unido a lo anterior, también aborda el concepto de aprendizaje cooperativo; Velásquez (2013) sostiene que es un tipo de metodología activa, en la que los estudiantes aprenden no tanto desde la transmisión de información por parte del docente, sino por las tareas que desarrollan y la reflexión que realizan sobre las mismas. Según este autor, se busca que los estudiantes alcancen objetivos académicos, pero también lograr metas sociales. Es por ello, que este tipo de aprendizaje se basa en el trabajo grupal pero estructurado, con base en principios que deben promover el aprendizaje de todos los estudiantes sin excepción.

Otra mirada que genera impacto en la investigación es el referido a la lúdica, entendida como una dimensión del desarrollo humano en donde se conjugan el placer, goce, la creatividad y el conocimiento. Ballesteros (2011) la define como aquella actividad la cual permite un ambiente de interacción accesible para el niño, partiendo de un rol, siguiendo de manera espontánea y libre una serie de normas previamente establecidas, potencializando así, habilidades que den paso a la exploración y experimentación, creando sensaciones, movimientos y promoviendo el avance en el aprendizaje.

Para finalizar, se aborda el concepto de juego, que de acuerdo con Huizinga (2013) es una de las actividades cotidianas del ser humano que se convierte en un ejercicio espontáneo que irradia cualidades nobles con reglas y límites provisionales, armónicamente aceptados por todos los actores involucrados. Por su parte, Baena (2016) señala que el juego, por su carácter espontáneo y placentero, se convierte en un excelente motor para promover la ejercitación natural del niño, fortaleciendo su desarrollo y crecimiento equilibrado de forma creativa, generando contextos que permitan mejorar sus hábitos comportamentales, cooperativos, de convivencia y el trabajo en equipo a través de la expresión natural de los sentimientos. 


\section{Metodología}

Por las características de la presente investigación, el contexto en el cual se desarrolla y atendiendo a todo lo propuesto en los capítulos precedentes, se ubica en el terreno de la investigación cualitativa. En efecto, Galeano (2004) sostiene que la investigación cualitativa trabaja con las cualidades de los seres humanos, los considera como productores de conocimiento, independientemente de su nivel educativo, de su condición socio económica y de su ubicación laboral. Este tipo de investigación en su estructura intima busca comprender a las personas - estudiantes- dentro de su propio marco de referencia, sus relaciones, es decir, en su ambiente natural; desde su vida misma, sus pensamientos y sentimientos (De La Espriella, 2013). Lo anterior, se sostiene en los planteamientos de Hernández, Fernández y Baptista (2014) quienes defienden la idea que la investigación con enfoque cualitativo lo que busca es comprender y profundizar los fenómenos, explorándolos desde la perspectiva de los participantes en un ambiente natural y en relación con el contexto.

El desarrollo de la investigación se abordó con la participación de los sujetos, en su contexto socio cultural, ello indujo a implementar la IAP, como método de investigación, entendida como un proceso dialéctico continuo en el que se analizan los hechos, se conceptualizan los problemas, se planifican y se ejecutan las acciones en procura de una transformación de los contextos, así como a los sujetos que hacen parte de los mismos (Calderón y López, s/f).

En este sentido, desde la IAP, se conoció a fondo la naturaleza del problema; la inmersión en el contexto permitió conocer realidades, problémicas y entender eventos que se dan en su interior. La producción de conocimiento por esta vía emerge a partir del diálogo con quienes construyen la realidad, que se entiende como propia de los sujetos que participan de la construcción de conocimiento social (Calderón y López, s/f). Por su parte, Fals y Anisur (1991) al desatacar el valor de la IAP, sostienen que en ella la búsqueda del conocimiento es el resultado de un proceso abierto de vida y de trabajo, una experiencia, una progresiva evolución hacia la transformación estructural de la sociedad y del sujeto como objetivos sucesivos y parcialmente coincidentes.

La población estuvo conformada por 139 estudiantes del grado cuarto de la IE San Clemente del municipio de Tierralta - Córdoba. La población estudiantil está diseminada en las 12 sedes con que cuenta la IE, para el caso del grado cuarto de primaria los estudiantes estaban distribuidos así: San Clemente, 33; Nuevo Tay, 42; Buenos Aires Tuis, 39; y Escuela Nueva Guarumal, 25. La unidad de análisis y sus participantes corresponde a los 42 estudiantes del grado cuarto de la sede Nuevo Tay; de los cuales 23 son niños y 19 niñas con edades entre los 10 y 12 años. Este curso fue elegido a conveniencia de uno de los investigadores puesto que es el lugar donde desarrolla su práctica profesional como docente de planta, adscrito a la IE. 


\section{Categorías de estudio}

Para el desarrollo de esta investigación, se han tenido en cuenta las siguientes categorías:

- Categoría conflicto escolar, en la cual se trabajan dimensiones como conflicto, convivencia, violencia escolar y resolución de conflictos, para las cuales se abordan diferentes tipos de indicadores.

- Categoría dispositivos lúdicos, en la cual se trabajan dimensiones como rol del docente, rol del estudiante, y ambiente escolar; para cada una de ellas, se trabajan diferentes tipos de indicadores.

\section{Fases o etapas de la investigación}

Las fases de esta investigación se describen partiendo del método IAP, a través de las cuales se desarrollaron las siguientes etapas:

Diagnóstico. En la primera etapa, se partió de la identificación del contexto social, comunitario y escolar de la IE San Clemente. Para lo cual, se recopilaron evidencias testimonios- mediante entrevistas con docentes y directivos. De igual forma se accedió a información documental asociada a la institución, entre ellas el Proyecto Educativo Institucional -PEl-, observador del alumno. Una vez todo lo anterior, se constituyó el grupo IAP, para este caso los estudiantes del grado cuarto de primaria y luego se da inicio al trabajo de campo. Previo a lo anterior, todos los participantes de la investigación -estudiantes y padres de familia- fueron informados sobre los procedimientos, objetivos y usos de la información recolectada en la investigación.

Construcción participativa - programación. Se conformó un grupo de discusión, del cual participaron diez estudiantes; con ellos, se debatió sobre el tema conflicto escolar y que soluciones darle al mismo. A partir de aquí, se inició la aplicación de los dispositivos lúdicopedagógico con la participación del docente investigador y el grupo de IAP, de acuerdo con el cronograma desarrollado en fases anteriores.

Recolección y análisis de la información. La recolección de información relevante para la investigación se desarrolló en dos momentos: uno en la fase diagnóstica, en donde se utilizaron técnicas como la entrevista semiestructurada, la observación participante, el grupo de discusión y la revisión documental; el segundo momento consistió en la aplicación de los DLP, que en sí mismos prestaron doble utilidad, una relacionada con el proceso de intervención-acción de la realidad -conflicto escolar-; y la otra, relacionada con la percepción de los estudiantes sobre la vivencia dada en el DLP. En consecuencia, los estudiantes luego de la vivencia, organizados en grupos de discusión reflexionan, reconstruyen instantes, expresan opiniones de lo experimentado; para luego dar paso a la aplicación de lo interiorizado, lo vivido, lo aprendido en el contexto escolar y familiar. Es decir, todo momento es constructivo, ya que se indaga al mismo tiempo que se interviene realidad. 
Para el análisis de información, se recurrió al análisis de contenido, técnica muy usada en ciencias sociales. En relación con el análisis de contenido Piñuel (2002) sostiene que:

Se suele llamar análisis de contenido al conjunto de procedimientos interpretativos de productos comunicativos -voces, mensajes, textos o discursos, reflexiones- que proceden de procesos singulares de comunicación previamente registrados, tienen por objeto elaborar y procesar datos relevantes sobre las condiciones mismas en que se han producido aquellos textos, o sobre las condiciones que puedan darse para su empleo posterior. (p.2)

Las voces, frases, párrafos escritos y reflexiones en la acción de estudiantes, docentes y padres de familia son los resultados a los cuales se le hizo el análisis de contenido, los cuales se cruzaron con las anotaciones del diario de campo y las reflexiones sobre la acción adelantadas por el investigador. Las reflexiones, individuales y colectivas derivadas de las vivencias, son consideradas rica fuente de información; son teorías, que develan las transformaciones en su comportamiento con los otros. Por ello, la investigación se identifica como emancipadora, puesto que los DLP facilitaron vivencias que contribuyeron a transformar las relaciones entre los participantes.

Lo anterior, evidencia que los resultados de la investigación no se dan a partir de explicaciones, ni datos derivados de aplicaciones de fórmulas o frecuencias. Ella es en sí misma, un ejercicio comprensivo de la vida de los sujetos -estudiantes-, en donde se reconoce entre si sus modos de vida, sus pensamientos, interacciones y la construcción de significados sobre su propia realidad, es decir, se captan los sutiles matices de las experiencias vitales (Vasilachis, 2006). Esto es a lo que Fals Borda llama -relaciones de intersubjetividad-, en donde no existen jerarquías de ninguna índole. Por consiguiente, los resultados de la investigación están en los sujetos, en este caso en los estudiantes que participaron de la experiencia, en las relaciones sociales que se generaron, en el proceso intersubjetivo, puesto que son los protagonistas de la formación y del aprendizaje que derivó en el cambio continuo. Por ello, sus voces, reflexiones y autorreflexiones fueron determinantes en esta investigación.

De igual forma, la reflexividad del investigador es sustancial a la hora de los resultados. En ese sentido, Flick (1998; citado por Vasilachis, 2006) sostiene que, a diferencia de otros métodos o enfoques de investigación, lo cualitativo toma a la comunicación del investigador sus reflexiones- con el campo y con sus miembros como parte explicita de la producción de conocimiento. Por consiguiente, la reflexión del investigador sobre sus acciones, observaciones, sentimientos, impresiones en el campo se transforman en datos, forman parte de la interpretación y son documentados en diarios de investigación o protocolos de contexto.

\section{Resultados y Discusión}

Los resultados de la investigación se presentan en función de los objetivos formulados: Implementar desde la Educación Física, Recreación y Deporte DLP encaminados a la transformación y resolución de conflictos de convivencia escolar en el grado cuarto de la IE San Clemente de Tierralta - Córdoba. Atendiendo a este objetivo, se formularon e 
implementaron DLP donde los estudiantes participantes experimentaron situaciones reales, en las cuales debían abrirse a sus compañeros, dialogar e interactuar entre ellos.

Cada DLP, -entendido como dispositivo de resistencia y potenciación- fue diseñado para provocar transformaciones, y construido con la participación de los sujetos de investigación estudiantes, docentes, padres de familia e investigadores- su diseño fue a la medida de las necesidades e intereses. Para fiabilidad y validez, todos los DLP diseñados y los retomados de otras fuentes, fueron sometidos a pilotaje previo y a juicio de expertos antes de ser implementados. En total fueron 50 que apostaban a diferentes propósitos, formas de interacción, creación, desenvolvimiento, dentro y fuera del aula. El pilotaje y juicio de expertos dejó como resultado la focalización de 13 de ellos, de los cuales se aplicaron nueve (Esquema 1); cada uno de ellos contaba con su respectivo objetivo asociado al tema a trabajar y las necesidades observadas.

Esquema 1. Descripción de los DLP implementados.

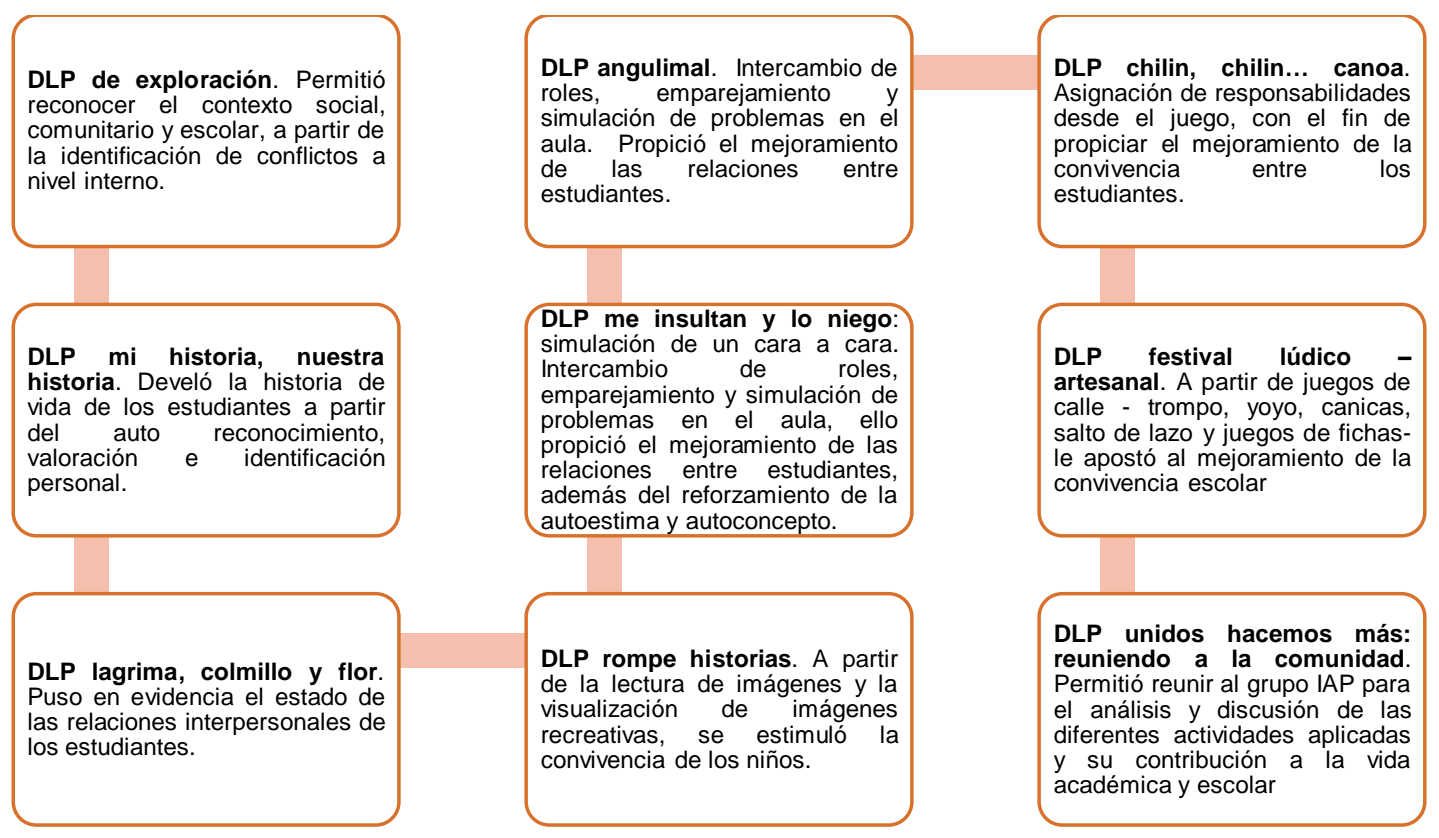

Fuente. Los Autores.

Los DLP desarrollados traen consigo además de la forma como aplicarlo, un espacio para las reflexiones, las voces de los participantes que se escuchan luego de la vivencia y que se enmarcan en tres aspectos:

\section{En la exploración y diagnóstico.}

En los actos de aula de los docentes, se evidenció que éstos se abordaban de manera lineal entre docente y estudiantes, era una especie de monologo, la relación docente estudiante se daban de forma mecánica y no generaban interacción con todo el grupo; la indagación sobre los presaberes, no eran prioridad y al final no se daba retroalimentación. Para los docentes los conflictos escolares pueden generarle diversos estados de ánimo, 
Hastings y Bham (2003; citados por Dobarro, Álvarez y Núñez, 2014) sostienen que estas situaciones pueden derivar en altos niveles de angustia y estrés; es más, pueden llevarle a la desmotivación laboral. La anterior tesis cobra sustento en el contexto de este estudio, ya que algunas voces de docentes al inicio decían: "estas estrategias tuyas son importantes; pero debes estar aplicándolas constantemente si se quiere notar cambios"; "de la noche a la mañana no se logra transformar esta situación"; "compañero, no busque meterse en camisa de 11 varas, cumpla con lo que le piden y ya". Todas ellas evidencian una desmotivación contagiosa en los docentes de la IE.

Luego entonces, si el docente es presa de la desmotivación, que se puede esperar del estudiante, el cual puede igualmente, por actitudes excluyentes y apáticas en el aula, mostrar trastornos del sueño, pérdida de apetito, ansiedad social, baja autoestima y autoconcepto negativo, mayor vulnerabilidad a la depresión, entre otras (Cerezo, 2008). En lo referente a la convivencia en el aula, se evidenció que los docentes solo tenían en cuenta las opiniones de los estudiantes que más participan en el desarrollo de la clase, excluyendo aquellos que observaban actitudes apáticas, distraídas o incluso desinterés.

Durante esta fase inicial, los padres de familia no asistían a las reuniones de acompañamiento de sus hijos; esto conllevaba al desconocimiento de la importancia del trabajo desarrollado en el aula y de hecho en la IE. Al respecto, Bohórquez (2008) sostiene que la realidad vivida el entorno familiar -relación padres e hijos- puede convertirse en un caldo de cultivo favorable para el desarrollo de tendencias violentas y antisociales. Por ello Ios DLP también le apostaron al acercamiento del padre al entorno escolar, esta realidad evidenciada llevó a significar el valor de la familia como piedra angular de la comunidad educativa.

\section{En la construcción participativa.}

Este proceso presentó dificultades al inicio, en la construcción de los DLP algunos estudiantes se mostraron apáticos, pasivos, desinteresados, -en cierta forma reflejaban la realidad del aula-. Sus intervenciones eran mínimas, se limitaban a escuchar al docente investigador. Algunos consideraban su participación como algo obligatorio, rutinario y aburrido. Uno de ellos expresó en voz alta "y esto pa que nos sirve", "esto no tiene que ver con la clase". No obstante, el acercamiento permanente, la retroalimentación constante de lo vivido, el buen trato, la palabra y el afecto, fueron venciendo los obstáculos poco a poco y modificando la actitud, manera de proceder y actuar de los estudiantes. Muchos se fueron integrando debido a su interés por conocer más sobre el proyecto. En efecto, por la recomendación constante que todos debían participar. Este fue un proceso gradual, en el que la motivación y búsqueda de relaciones pacíficas fue una constante.

La participación de algunos padres y docentes en este momento de la investigación fue determinante. Poco a poco, se fue transformando la división en unión, el conflicto en una búsqueda de paz y la formación en valores. Así los DLP se diseñaron en conjunto con los actores. Para que la acción del docente surta efecto, es necesario que los padres de familia y 
la institución reconozcan su responsabilidad en el proceso educativo de los niños, niñas y jóvenes, y no dejen solo al maestro. Sobre lo anterior, Domínguez (2010) sostiene que la escuela debe reconocer y aceptar la importancia de la participación y la colaboración de los padres en la educación de los niños y niñas, para tales propósitos se necesita de una relación cordial entre docente y padres para que los docentes puedan realizar su función de manera efectiva y completa.

\section{En la aplicación de los DLP.}

La implementación de los DLP permitió acercamientos entre los actores, los cambios de actitud se fueron dando entre ellos, en su manera de proceder. El interés por el proyecto fue aumentando y querían saber más sobre su desarrollo. Expresiones recogidas en las guías de observación y en las reflexiones de los participantes dan cuenta de esto. Algunas voces de docentes decían: "compañero va por buen camino, siga así"; "ha logrado sembrar una semilla"; "profesor, poco a poco ha motivado a los niños a salir adelante, a interactuar con los demás"; "sus implementaciones están acordes a las necesidades de los chicos, pero hay que hacer más".

Desde lo institucional, algunos directivos docentes manifestaron su sentir en una reflexión con el colectivo docente: "en la institución son muy pocas las personas que se han propuesto intervenir en las situaciones que se viven acá, la mayoría solo cumple con sus horarios y listo, pero con su trabajo ha demostrado marcar un poco la diferencia". "Los padres de familia han manifestado su interés por participar y estar más pendientes de los procesos escolares de sus hijos, al respecto, preguntan constantemente cómo se debe trabajar el conflicto o qué estrategias pueden implementarse". Sobre el deber ser del maestro, Sosa, Sosa y González (2010) consideran que el buen maestro debe conocer bien a sus estudiantes, darles el afecto requerido en medio de la diversidad e inclusión, hacer de su trabajo un acto educativo humano, mantenerse informado e informar a los estudiantes sobre lo que es nuevo en la ciencia, cuáles son las experiencias avanzadas; en síntesis, debe crear las condiciones necesarias para fomentar el desarrollo de conocimientos, capacidades y habilidades en sus estudiantes, todo lo anterior, de forma tal que les permitan enfrentar diversas situaciones y saber buscar las vías de solución a cada una.

Los DLP en cada una de sus sesiones, siempre abordó el conflicto desde una perspectiva positiva, Jares (1997) citado por Pérez y Gutiérrez (2016), sostiene que la necesidad de afrontar y resolver los conflictos se centra en la optimización y funcionamiento del grupo, de restablecer o perfeccionar la comunicación y las relaciones humanas a través del entendimiento de las subjetividades personales. Por consiguiente, las causas de los conflictos se atribuyen a problemas de percepción individual o bien, a una deficiente comunicación interpersonal; motivos que, efectivamente, pueden provocar conflictos, pero que ni agotan esas posibles causas ni los explican en toda su complejidad.

Las historias de vida (HV) de los estudiantes develaron tramas de significados enmarcados en sus relaciones y problemas, así como las formas de promover, mediar, discutir 
y dar soluciones a los conflictos que a diario viven en su familia y la escuela. En ese sentido, la HV es un instrumento para interpretar información, útil para el investigador como medio para la generación de diálogos abiertos con los estudiantes. En relación con lo anterior, Páez (2017) sostiene que en la escuela es vital la búsqueda de diálogos y conversaciones que posibiliten el conocimiento compartido, la práctica del saber cómo trasformación de realidades, la pedagogía horizontal como escenario de aprendizaje colectivo y la inclusión del docente como factor de tensión en la escuela. La interpretación de las vivencias de los estudiantes permitió evidenciar dificultades de aprendizaje, de convivencia, autoestima y autoconcepto. Esta intervención le permitió al conflicto la alternativa de catalizarse, de resolverse desde la óptica de los otros (Páez,2017).

El proceso investigativo develó el concepto de conflicto que tienen los estudiantes. Para algunos de ellos el conflicto se relaciona con acciones como robar y asesinar, peleas individuales o grupales, o se relaciona con el accionar de bandas criminales y guerrilla. En este sentido, se puede apreciar cómo algunos de estos estudiantes relacionan al conflicto con violencia, pelea, matanza y robo. Por lo cual, lo contextualizan en un escenario negativo donde predomina la agresión del uno hacia el otro o de un grupo de personas hacia otro. Sumado a esto, otros estudiantes incluso mencionan bandas, guerrilla y también a la violencia sexual. Esto se relaciona con el concepto emitido por Julien Freund (1983) quien define al conflicto como un choque intencional en el que se deja en manifiesto una intención hostil, generalmente acerca de un derecho, buscando romper la resistencia del otro a través de la violencia.

Cabe destacar que en gran mayoría los estudiantes del grado cuarto que participaron en el grupo de discusión coincidieron en afirmar que los conflictos sí son necesarios. Independientemente de la ausencia de justificación que ellos le otorgan a su respuesta, teniendo en cuenta el escenario de relaciones interpersonales que se desarrollan en las instituciones educativas entre los estudiantes, esta afirmación tiene mucha relación con los argumentos de Vinyamata (1999) quién señala que existen conflictos de carácter interpersonal los cuales son necesarios e inevitables, que se desarrollan en cualquier esfera de la realidad social posibilitando la construcción de nuevas y constantes relaciones sociales.

Al cuestionar a los niños y niñas sobre porqué surgen los conflictos, señalaron que generalmente, se dan entre dos personas. También afirman que se presentan porque un estudiante provoca al otro hasta lograr desencadenar una discordia. Se habla de que, al surgir el conflicto, este puede culminar en la matanza de un individuo hacia el otro. Algunos estudiantes ven como raíz del conflicto a la provocación permanente de un individuo hacia el otro, lo cual desencadena situaciones de agresión y violencia. Esta apreciación se relaciona con el concepto de provocación emitido por Porto y Gardey (2013), quienes señalan que este término adquiere distintos significados dependiendo del contexto y que para el caso de las relaciones interpersonales el concepto que mejor se acomoda es el de generar una reacción sentimental o una emoción, la cual para el presente caso se trataría de una negativa.

Al indagar sobre la consideración que se tiene sobre los conflictos que se presentan en la institución, señalaron que son normales, fáciles de sobrellevar y solucionar. Frente al tema del nivel de complejidad de los conflictos escolares que suelen presentarse, aparece el tema 
del desquite a cuál es percibido por los estudiantes constantemente en la presentación de situaciones de violencia en la institución. Del mismo modo, Freud (1893; citado por Ramos, 1992), señala que, en muchas ocasiones cuando el ultraje es devuelto se logran evitar episodios traumáticos para la persona afectada. Ahora bien, teniendo en cuenta la vinculación de los padres para comprender e intervenir la problemática de los estudiantes involucrados en situaciones de violencia, Bohórquez (2008) argumenta que el entorno familiar puede convertirse en un caldo de cultivo favorable para el desarrollo de tendencias violentas y antisociales. Tal como lo expresa Domingo (2008), la escuela debe aceptar la importancia de la participación y la colaboración de los padres en la educación de los niños.

Por otra parte, las interacciones y los dispositivos se desarrollaron de manera consecuente con el fin de realizar posibles ajustes durante su aplicación. Este aspecto puede ser considerado como un valor agregado e importante en la consecución de los objetivos de la investigación, ya que se empezó a involucrar el trabajo colaborativo, la estimulación de la participación en las actividades, entre otros aspectos pedagógicos. En consecuencia, se tuvo coherencia en cuanto no se realizaban exclusivamente de modo individual o solo en el aula de clase. Esto incidió en mejorar las relaciones de convivencia ya que los niños y niñas podían aplicar conocimientos generales o sus experiencias en los elementos trabajados, obviamente, desde la puesta en marcha de la investigación, para luego ser compartidas con su grupo, lo cual la hacían más interesante para ellos

En lo concerniente a la transversalidad -otro valor agregado dentro de la propuesta-, se evidenció con la puesta en marcha el trabajo en equipo de los docentes, fue propicio dentro de la planeación estratégica, la aplicación de los DLP y en la implementación de estos dentro de las metodologías utilizadas. Por ello, se hace pertinente reconocer que dentro del clima formativo se deben tener en cuenta los intereses y motivaciones de los niños y niñas, todo con el fin de realizar una verdadera transformación pedagógica y social, en tal sentido, es posible afirmar que los dispositivos tuvieron coherencia. Otro aspecto evidenciado fue el trabajo colaborativo, ya que no se realizaban de manera individual o solamente en el área de instrucción; las propuestas se desarrollaron a partir de temáticas que se abordaban desde las diferentes asignaciones, teniendo en cuenta los objetivos de formación.

\section{Conclusión}

Al realizar un balance de los DLP implementados con los estudiantes del grado cuarto de la IE San Clemente de Tierralta en el departamento Córdoba, es posible afirmar que se logró una intervención que derivó en transformaciones, puesto que el proceso de formación se constituyó en una relación positiva, activa y reflexiva entre todos los participantes del proceso escolar, generando con esto la construcción, diseño e implementación de dispositivos que permitieran superar conflictos de convivencia en el curso mencionado a partir de la integración de la Educación Física, Recreación y Deportes. 
Una característica distintiva de este estudio es el trabajo interactivo y la interlocución permanente con los otros participantes, quienes aportaron conocimientos diferentes a los de la academia, pero que de todas maneras fueron conocimientos válidos que ayudaron a comprender la realidad. Es de resaltar, que uno de los elementos que permitió este proceso, fue la implicación de aspectos éticos y morales como los valores, gracias a los cuales se hizo énfasis en la necesidad de ser mejores personas, más competentes o responsables con sus conocimientos y la manera en que los utilizan; sobre todo, al momento de llevar a cabo procesos de convivencia o de desenvolvimiento en su contexto. Se evidencia entonces, la trascendencia de realizar y aplicar los DLP ya que al finalizar el proceso de instrucción en el grupo al cual se aplicó, se observó una disminución de los factores antes mencionados como indisciplina, peleas, ausentismo y poco aprendizaje de las temáticas estudiadas. Ahora bien, al momento de dar por finalizada la implementación de los DLP, los directivos, docentes, estudiantes y la comunidad en general de la IE San Clemente, logró vincularse a los procesos de enseñanza y aprendizaje, a través de la inter y transversalidad, lo que permitió fortalecer los procesos de aprendizaje y las prácticas de enseñanza en el aula.

Por todo lo planteado con anterioridad, se tiene que el fortalecimiento de la convivencia educativa y el tratamiento de conflictos escolares en los estudiantes, debe ser una prioridad en la educación, conduciendo a una formación en competencias cuyos conocimientos y prácticas incluyan también el saber convivir y relacionarse con el entorno que los rodea. El reto de la educación es preparar a los niños y jóvenes a ser personas autónomas, pensantes, responsables, productivas, ante todo con un gran sentido humanista y del respeto por las diferencias con sus semejantes, de ahí que los estudiantes necesiten desarrollar prácticas sanas de convivencia que les permita relacionarse armónicamente con sus compañeros, docentes y directivos, fomentando una interacción significativa y un enriquecimiento continuo de las competencias comunicativas. Ahora bien, es evidente que educar para la sana convivencia implica que todas las áreas del saber se sientan involucradas, directa 0 indirectamente con esta problemática, sobre todo teniendo en cuenta que las apariciones de situaciones conflictivas en las aulas de clases ha resultado ser una situación que cada vez toma más relevancia y se torna más difícil de tratar.

\section{Referencias}

Baena, A. (2016). El juego motor como actividad física organizada en la enseñanza y la recreación. Revista Digital de Educación Física, Vol. 7, $N^{\circ} 38$

Ballesteros, O. (2011). La lúdica como estrategia didáctica para el desarrollo de competencias científicas. (Tesis Maestría). Colombia: Universidad Nacional de Colombia.

Bohórquez, E. (2008). El papel de la familia en la violencia escolar. Revista ECO. Vol. 1, No. 4. Recuperado en: http://www.cepazahar.org/eco/n4

Bravo, I. y Herrera, L. (2011). Convivencia escolar en educación primaria. Las habilidades sociales del alumno como variable moduladora. (Tesis Maestría). Disponible en: Dedica: Revista de educación y humanidades, p.p. 173-212. 
Calderón, I. (2011). La mediación en la resolución de conflictos en los contextos escolares, Revista Acción pedagógica.

Calderón, J. \& López, D. (s/f). Orlando Fals Borda y la investigación acción participativa: aportes en el proceso de formación para la transformación. I Encuentro hacia una Pedagogía Emancipatoria en Nuestra América. Recuperado en: https://www.javeriana.edu.co/blogs/boviedo/files/pedagogc3adas-emanIc3b3pez-cardona-y-calderc3b3n.pdf

Camilloni, A. (2013). Las metáforas conceptuales en el discurso pedagógico. Conferencia de apertura de las VII Jornadas Nacionales sobre la Formación del Profesorado: Narrativa (s), Práctica (s) e Investigación (es). Mar de la Plata, Argentina.

Castillo, L. (2011). El acoso escolar. De las causas, origen y manifestaciones a la pregunta por el sentido que le otorgan los actores. Magis, Revista Internacional de Investigación en Educación.

Cerezo, F. (2008). Acoso escolar. Efectos del bullying. Boletín de la Sociedad de Pediatría de Asturias, Cantabria, Castilla y León. Recuperado en: https://www.sccalp.org/documents/0000/0147/BolPediatr2008_48_353-358.pdf

Decreto 1965. (2013). Diario Oficial. N. 48910. Recuperado en: http://www.suinjuriscol.gov.co/viewDocument.asp?ruta=Decretos/1378136

De la Espriella, F. (2013). Historia de vida de Lely Luz Flórez Meza: boxeadora colombiana excampeona mundial de la categoría wélter junior del Consejo Mundial de Boxeo (CMB). Educación Física y Deporte. $\mathrm{N}^{\circ} 32$, Vol. $1 . \quad$ Pág., $1301 \quad$ - $1314 . \quad$ Recuperado en: https://aprendeenlinea.udea.edu.co/revistas/index.php/educacionfisicaydeporte/article/download/1 $\underline{6512 / 15586}$

Dobarro, A., Álvarez, D. y Núñez, J. (2014). Instrumentos para evaluar la violencia escolar. International Journal of Developmental and Educational Psychology. INFAD Revista de Psicología. Recuperado en: http://www.infad.eu/RevistalNFAD/OJS/index.php/IJODAEP/article/download/710/644

Domingo, J. (2008). El aprendizaje cooperativo. Cuadernos de trabajo social. Vol. 21, p.p. 231-246

Domínguez, S. (2010). La Educación, cosa de dos: La escuela y la familia. Andalucía: Temas para la educación. Recuperado en: https://www.feandalucia.ccoo.es/docu/p5sd7214.pdf

Fals, O. y Anisur, M. (1991). Acción y conocimiento: Rompiendo el monopolio con la IAP. Bogotá: Rahman.

Fisas, V. (2010). Introducción a los procesos de paz. Quaderns de construcción de Pau. Escola de cultura de Pau. (ECP). N 12, pág. 30.

Freund, J. (1983), Sociologie du conflicto, Presses Universitaires de France, París

Gairín, J., Armengol, C. \& Silva, B. (2013). El bullying escolar. Consideraciones organizativas y estrategias para la intervención.

Galeano, M. E. (2004). Diseño de proyectos en la investigación cualitativa. Medellín: Fondo Editorial Universidad Eafit.

Galtung, J. (1998) Tras la violencia, R: Reconstrucción, Reconciliación, Resolución. Afrontando los efectos visibles e invisibles de la guerra y la violencia. Bilbao: Bakeaz Gernika Gogoratuz.

García, M. (2015). El conflicto y sus tipos en el ámbito escolar. Revista Arista Digital, $N^{\circ}$ 52. Disponible en: http://www.afapna.es/web/aristadigital 
Guber, R. (2001). La etnografía: método, campo y reflexividad. Bogotá: Ed. Norma.

Guzmán, E., Muñoz, J. \& Preciado, A. (2012). La convivencia escolar: una mirada desde la diversidad cultural. Facultad de Ciencias Sociales y Humana. Universidad de Manizales.

Hernández, R.; Fernández, C. y Baptista P. (2014). Metodología de la Investigación 6ta Edición. Editorial McGrawHill Interamericana Editores S.A. México.

Huizinga, J. (2013). El elemento lúdico en la cultura: Homo Ludens. Recuperado de: https://perjam24.wordpress.com/2013/11/09/huizinga-johan-1987-el-elemento-ludico-en-la-culturahomo-ludens/

Merayo, M. (2013). Acoso escolar. Confederación española de asociaciones de padres y madres de alumnos.

Páez, A. (2017). La historieta de vida como resolución del conflicto escolar. Cambios y permanencias. Recuperado en: https://revistas.uis.edu.co/index.php/revistacyp/article/view/7831/8003

Pérez, E., \& Gutiérrez, D. (2016). El conflicto en las instituciones escolares. Recuperado en: http://www.redalyc.org/articulo.oa?id=46146811010

Piñuel, J. (2002). Epistemología y técnicas del análisis de contenido. Facultad de CC. De la Información. Universidad Complutense de Madrid. Recuperado en: https://www.ucm.es/data/cont/docs/268-201307-29-Pinuel Raigada AnalisisContenido 2002 EstudiosSociolinguisticaUVigo.pdf

Porto, J.; y Gardey, A. (2013). Definición de provocación. Obtenido de http://definicion.de/procedimientoadministrativo/prov_

Ramos, R. (1992). Tiempo y sociedad. Madrid, CIS - Siglo XXI.

Sosa, J., Sosa, L., \& González, M. (2010). La pedagogía del docente universitario en la dirección del proceso de formación integral de los estudiantes. Cuaderno de educación y desarrollo. Recuperado de: http://www.eumed.net/rev/ced/15/ssl.htm

Vasilachis, I. (2006). Estrategias de investigación cualitativa. Barcelona: Ed. Gedisa. Recuperado en: http://jbposgrado.org/icuali/investigacion\%20cualitativa.pdf

Velásquez C. (2013). Análisis de la implementación del aprendizaje cooperativo durante la escolarización obligatoria en el área de educación física. (Tesis doctoral). Universidad de Valladolid, Facultad de Educación y Trabajo Social. España, Valladolid.

Vinyamata, E. (1999). Manual de prevención y resolución de conflictos: conciliación, mediación, negociación, Barcelona: Ariel Practicum.

Vinyamata, E. (2015). Conflictología. Revista de paz y conflictos. (Vol. 8. $\mathrm{N}^{\circ}$ 1.). Recuperado en: https://dialnet.unirioja.es/descarga/articulo/5117130.pdf. 